\title{
Incentives for Infrastructure Deployment by Over-the-Top Service Providers in a Mobile Network: A Cooperative Game Theory Model
}

\author{
Jacek Kibiłda*, Francesco Malandrino ${ }^{\dagger}$ and Luiz A. DaSilva* \\ *CONNECT, Trinity College Dublin, Ireland, e-mail: \{kibildj,dasilval $\} @$ tcd.ie \\ ${ }^{\dagger}$ Politecnico di Torino, Torino, Italy, e-mail: malandrino@tlc.polito.it
}

\begin{abstract}
The success of smartphones has encouraged overthe-top service providers to seek ways in which they can have more control over the wireless service offered to their users. Google Project $\mathrm{Fi}$ is an example for this type of action, where control over the wireless service is achieved by either deploying own wireless infrastructure or entering into service level agreements with mobile network operators. Following this example, we construct a game theoretic model for the interaction between mobile network operators and over-the-top service providers and assess how the spatial distribution of mobile demand impacts the outcomes of cooperation, unevenly affecting the utilities achieved by the two parties. We also show how the cost of fixed infrastructure deployed by the mobile operator, if too high, may render cooperation between the two parties ineffective.
\end{abstract}

\section{INTRODUCTION}

The new technological challenge ahead for mobile network operators (MNO) is to meet a 1000-fold increase in demand for mobile capacity [1]. However, meeting this challenge requires that MNOs face the hard facts of ever-increasing infrastructure costs [2] and popularity of over-the-top services which "extract revenue that would otherwise be generated by the MNO from traditional voice and data services" [3]. Ensuring that mobile networks provide adequate capacity for over-the-top services is of particular concern for over-the-top providers (OTT), such as Google, Skype or Netflix, which rely on mobile networks to offer their services and content to their mobile users. Taking Google as an example, we see an increasing push among the OTTs to assert their role in shaping how mobile networks are accessed and deployed. The recently announced Google Project Fi establishes a new type of a player in the mobile market, namely one that is simultaneously an over-the-top service provider, an infrastructure provider and a mobile virtual operator. Subscription to Fi provides wireless access using unlicensed $\mathrm{WiFi}$ infrastructure, contracted or deployed by Google, or, if WiFi is unavailable, the cellular network of T-Mobile or Sprint. Another example is Google Free Zone, launched in some developing countries, which allows mobile subscribers to access their Google social media profile or email account without having to pay data fees to their MNO.

The business-technological decisions OTTs, like Google, make are changing the way mobile networks are deployed, accessed and controlled. In this paper we analyze a simple model of a business-technological relationship between an OTT and an MNO. We consider that the OTT is looking for ways to ensure a certain quality of experience to its mobile subscribers. In order to do so the OTT has either the choice of deploying its own radio access infrastructure, similarly to Google's WiFi deployments made in some Northern American cities, or entering into a service level agreement (SLA) with an $M N O$, following the Google Project Fi example. In the former case the OTT bears full costs of the required unlicensed radio access infrastructure, while in the latter case it pays recurring charges to the $\mathrm{MNO}$. The $\mathrm{MNO}$, in turn, can invest this additional revenue in the densification of its network, for example, by deploying small cells.

The decisions made by both OTTs and MNOs are not independent, as whatever the decision one party makes, it affects the outcome observed by the other party. A natural tool to model this type of interaction is game theory, and cooperative game theory, in particular. In cooperative games players typically have an incentive to enter into a cooperative agreement, yet they may have conflicting views on the specifics of such an agreement. In our case the OTT requires radio access infrastructure, which the MNO already has, while the MNO seeks additional revenues to cope with the increasing infrastructure costs. At the same time the OTT would like to utilize the infrastructure as cheaply as possible, while the MNO collects revenues from the usage of its infrastructure and charges the OTT (or its subscribers) accordingly.

Our main contributions can be summarized as follows. In this paper we propose the aforementioned model of cooperation between an OTT and MNO, for which we define the corresponding utility space and the non-cooperative point related to the Nash equilibrium. Then, using the Nash bargaining framework [4], we derive an analytical expression for the Nash bargaining solution (NBS) of the corresponding game. What we show is that the spatial distribution of mobile demand has a significant impact on the outcome of cooperation, and that it unevenly affects the utilities achieved by OTTs and MNOs. Moreover, the cost of MNO's infrastructure, if excessively high, may render cooperation between an OTT and MNO ineffective.

\section{BACKGROUND AND RELATED WORK}

In [3] we have proposed a vision of future wireless networks that relies on virtualization and pooling (aggregation) of resources, such as cellular base stations, frequency spectrum or household access points, belonging to multiple infrastructure 
providers to enable dynamic orchestration of wireless networks crafted for a particular service offered by an OTT. We view the resulting networks as service-driven, since over-the-top services affect the way these networks are managed and deployed [5], [6]. Indeed, an increasing volume of research recognizes provisioning of over-the-top services as the key feature of the next generation mobile networks: in [7] $5 \mathrm{G}$ network management architecture and management interfaces are defined to provide support for over-the-top services, while [8] proposes a control plane architecture that supports dynamic SLAs.

In this paper we are concerned with modelling the interaction between OTTs, which offer over-the-top services, and MNOs, which provide (wireless) infrastructure. The majority of models relevant to our work were studied in connection with the "net neutrality" discussion. In [9] we find a summary of various possible frameworks for the interaction between OTTs and MNOs. These interactions can be classified based on the pricing regime applied, i.e., one-sided, where no additional fees are collected from service providers, or twosided, where service providers are charged according to the terms of bilateral SLAs with infrastructure providers. Among one-sided pricing models we can find the status quo model, where best effort traffic of various OTTs is subject to traffic engineering techniques which limit certain types of services, for example, by blocking peer-to-peer traffic, or the strict net neutrality model, where network operators apply nondiscriminatory traffic management, yet they cap the available capacity on a per user basis, or the user-tiering model, where the network operator charges users for different quality of service (QoS) levels delivered. Two-sided pricing involves OTTs paying either a guaranteed traffic fee corresponding to the guaranteed QoS level (service provider-tiering model) or a termination fee which depends on the volume of OTT traffic that terminates in the MNO network (termination fee model). Leaving aside the political (and business) implications, each of these approaches has its strengths and weaknesses, and can possibly be combined into more sophisticated pricing schemes, for example, the channel quality-based pricing [10]. We construct our model based on two-sided pricing and service provider-tiering. This and similar pricing schemes have been applied widely to study the impact of regulation on the wired networks and the Internet market (see [11]-[13]).

We model the OTT-MNO interaction using axiomatic bargaining. In the axiomatic bargaining framework two players with conflicting objectives seek to establish an agreement that will lead to a more favourable outcome than the outcome they would be able to achieve without negotiation. Axiomatic bargaining ignores the process of negotiations of the cooperative agreement (i.e., the bargaining process) and focuses on the outcome of cooperation. This outcome is defined through a set of properties (axioms) and the decisions that players make are a direct consequence of those properties (see [4]). In practice, that means if an OTT and MNO decide to cooperate they sign an SLA which defines the terms of cooperation, i.e., the price the MNO charges the OTT and the volume of OTT demand the MNO will serve through its network. The properties of the outcome are selected so as to represent rational terms of cooperation, and are well-defined, for example, for the Nash bargaining solution [4]. We should also note that our model can be easily generalized to the case of multiple OTTs with different services, which form a federation to provide an integrated service. Let us note also that the OTT-MNO interaction may also be projected onto a more conventional relationship between an entrant MNO and incumbent MNO, in which case the former may enter into a national roaming agreement with the latter [14].

Axiomatic bargaining has been applied in wireless research to date to study rate regions of interference channels [15], dynamic spectrum access [16], resource allocation in singleoperator networks [17], resource allocation in shared networks [18], or cooperation among wireless service providers [19].

\section{OTT-MNO INTERACTION MODEL}

The players in our model are the OTT (or an aggregate of OTTs) and the MNO. The OTT has to decide whether to build its own infrastructure or to let the MNO serve its demand. The decision is not binary, as the OTT may decide to serve a fraction of its demand using its own infrastructure, while the remaining demand will be served by the MNO and is subject to a per unit of served traffic charge decided by the MNO. Effectively, the MNO may need to expand its network to host the subscriber base of the OTT with the required quality, and cover the resulting costs. The decisions made by the OTT and MNO are coupled, i.e., the OTT will decide to serve a certain portion of its demand through the MNO network only if the price per traffic unit is acceptable, while the MNO will set the price to a certain level in response to the volume of the offloaded traffic, and considering factors, such as the costs of deployment and operation of its own infrastructure.

\section{A. Game model}

We model the interaction between an OTT and MNO in the following way. The OTT has to provide service to a set of spatially distributed mobile demand points $\mathcal{S} \subset \mathbb{R}^{2}$. Each demand point is associated with some volume of demand $r \in$ $\mathbb{N}$, and the marked set of demand points is denoted as $\hat{\mathcal{S}}=$ $\left\{\left(s, r_{s}\right)\right\}$, with the total demand (per OTT) of $R=\sum_{r \in \hat{\mathcal{S}}} r_{s}$. In order to provide service to its demand points the OTT may either deploy its own radio access infrastructure (at a unit cost $c_{o}$ ) or enter into an SLA with the MNO. In the latter case the OTT has to pay the per traffic unit charge decided by the MNO for the demand it decides to serve with the MNO network. The MNO serves the OTT's demand using the existing LTE macrocell infrastructure, which bears no additional deployment costs, or, if that is not possible (due to lack of coverage or capacity), using newly deployed small cell infrastructure at a unit cost $c_{m}$. Additionally, we denote $Q$ as the revenue received by the OTT from its mobile subscribers. Since in this paper we are interested in studying the OTT-MNO interaction we assume that $Q$ is fixed and no further assumption about the OTT subscribers needs to be made.

From the system's description we formulate the game model as follows: $\mathcal{N}=\{$ OTT, MNO $\}$ is the set of players; $[0,1] \times[0,1]$ is the action space, where $\theta \in[0,1]$ is the decision of the OTT, i.e., the fraction of the demand to be served by its network, in case it decides to deploy its own infrastructure (while the rest gets served by the MNO network), and $\beta \in[0,1]$ is the decision of the MNO, i.e., the per traffic unit charge (normalized as described in the following 
paragraph); and $\Omega=\left(u_{o}(\beta, \theta), u_{m}(\beta, \theta)\right)$ is the utility space, with $u_{o}(\beta, \theta)$ denoting the utility obtained by the OTT and $u_{m}(\beta, \theta)$ denoting the utility obtained by the MNO.

Up to now we have defined traffic-related measure $r_{s}$ and various cost-related parameters, yet we have not discussed their representation. First, we consider all of our economic parameters to be per unit of traffic served during a certain time frame. This means, for example, amortizing the deployment cost of a base station over an appropriate period. This approach allows us to construct utility functions that combine revenues and costs related to the user traffic with the costs of base station deployments. Second, all our economic parameters are normalized against $c_{o}$, which allows us to simplify the utility functions used and make the model independent of particular choices of monetary values. An additional implication of this assumption is that the traffic charge $\beta$, when normalized against $c_{o}$, can be limited to $[0,1]$. The rationale is as follows: when the cost of offloading a unit of traffic is higher than the cost of deploying a base station per unit of traffic, then the OTT is more likely to deploy a base station than to offload its traffic. Ultimately, the traffic can be represented as bytes, packets, flows, etc., with the selection of the traffic unit being independent from our model.

\section{B. Utility space}

We define $u_{o}(\beta, \theta)$ as follows:

$$
u_{o}(\beta, \theta)=-m_{o}(\theta, \hat{\mathcal{S}})-\beta(1-\theta) R+Q,
$$

where $m_{o}(\theta, \hat{\mathcal{S}})$ denotes the number of base stations to be built by the OTT, given $\theta$ and demand $\hat{\mathcal{S}}$. The rationale for this expression of the utility is straight-forward - the OTT has to pay for any new infrastructure it decides to build and/or pay the traffic fee for the demand served by the MNO network. In return the OTT receives revenue from its subscribers either directly, as part of a subscription fee, or indirectly, from customized advertising.

The MNO obtains the following utility:

$$
u_{m}(\beta, \theta)=-c_{m} m_{m}\left(\hat{\mathcal{S}}_{\text {res }}\right)+\beta(1-\theta) R,
$$

where $m_{m}\left(\hat{\mathcal{S}}_{\text {res }}\right)$ denotes the number of base stations to be built by the MNO to serve the demand $\hat{\mathcal{S}}_{\text {res }}$, where $\hat{\mathcal{S}}_{\text {res }}=f(\theta, \hat{\mathcal{S}})$, with $f:[0,1] \times\left(\mathbb{R}^{2}, \mathbb{N}\right) \rightarrow\left(\mathbb{R}^{2}, \mathbb{N}\right)$, is the residue of the OTT's demand not served by the OTT's infrastructure, which we discuss in the following paragraph. The MNO pays all the deployment costs associated with the deployment of additional base stations (we assume that the existing infrastructure bears no deployment costs) required to satisfy the residual demand, while at the same time receiving revenue proportional to the served traffic.

The utilities of both the OTT and MNO depend on the number of new base stations required to satisfy the demand, i.e., $m_{o}(\theta, \hat{\mathcal{S}})$ and $m_{m}\left(\hat{\mathcal{S}}_{\text {res }}\right)$. In its generality the problem of deploying wireless access infrastructure is a complicated one consisting of various planning stages (see [20]) and requiring various smart ways to find solutions to NP-hard problems (see [21]). Since our goal is to study the relationship between an OTT and MNO, instead of directly modelling the deployment decisions (i.e., whether a base stations should be built in some given location), we are interested in aggregate decisions, such as "How many base stations are required given certain volume of spatially distributed demand?" In other words, we seek a mapping between the demand and a counting measure of the number of base stations deployed to serve the demand, i.e., $m_{o}(\theta, \hat{\mathcal{S}})$ and $m_{m}\left(\hat{\mathcal{S}}_{\text {res }}\right)$, which hereafter we will refer to as the deployment function. We determine the deployment function for both the OTT and MNO empirically by applying the following procedure.

First, we generate the set of demand points $\mathcal{S}$ as the realizations of the Gauss-Poisson process [22], with the parameter cluster probability that allows us to vary the level of clustering in $\mathcal{S}$. Subsequently, we extrapolate real-world call-detail record information from an Irish mobile operator into a continuous demand field, which we sample using points from $\mathcal{S}$ to obtain $\hat{\mathcal{S}}$. Effectively, we construct a demand model with real demand volume information and varying level of clustering, which we control through the cluster probability. We use $\mathcal{\mathcal { S }}$ to feed an optimization model which minimizes the deployment cost, given that $\theta$ fraction of demand $\hat{\mathcal{S}}$ needs to be served using OTT's infrastructure. The outcome of the model is a set of WiFi access points which the OTT has to deploy, and the residual demand $\hat{\mathcal{S}}_{\text {res }}$, which will be offloaded to the MNO. To find the set of new infrastructure required by the MNO to provide coverage to $\hat{\mathcal{S}}_{\text {res }}$ we use an optimization model which minimizes the deployment cost, subject to covering all of $\hat{\mathcal{S}}_{\text {res }}$ with either new small cell or existing macrocell infrastructure (in both cases we assume single-antenna LTE system). This optimization model also considers the requirement that the MNO must serve its own subscribers' demand. Now, for each value of cluster probability, we obtain a curve that represents the number of base stations required as a function of $\theta$, to which we can fit some well-known functions. We obtained the most satisfactory fits with the quadratic function of the form $a_{0}+a_{1} \theta+a_{2} \theta^{2}$, where the coefficients vary with the cluster probability (the obtained coefficients are given in Table I). We will use $a_{\{0,1,2\}}$ to denote the coefficients of $m_{o}(\theta, \hat{\mathcal{S}})$ and $b_{\{0,1,2\}}$ to denote the coefficients of $m_{m}\left(\hat{\mathcal{S}}_{\text {res }}\right)$. Since our demand model $\mathcal{S}$ is stochastic, the deployment function should be interpreted as the average number of base stations to be built for a given clustering level of spatially distributed demand.

The point of this whole procedure was to determine a closed-form representation for the deployment functions, such that some level of analytical tractability is achieved. Yet, our utility space $\Omega$, resulting from the postulated utility functions, is not convex. However, if correlated mixed strategies are permitted, then the considered utility space becomes convex [4]. An example utility space for our model is illustrated in Fig. 1. It was obtained from drawing a convex hull over utility pairs calculated for the action space varied over $[0,1] \times[0,1]$. The point marked as NE denotes the Nash equilibrium, which corresponds to a non-cooperative solution to our game model, while the NBS corresponds to the cooperative solution, which we find analytically in Sec. IV.

\section{Disagreement point}

In any cooperative game we need to define the disagreement point, which is the point of minimal utility (payoff) expected by each of the players if bargaining (negotiations) 


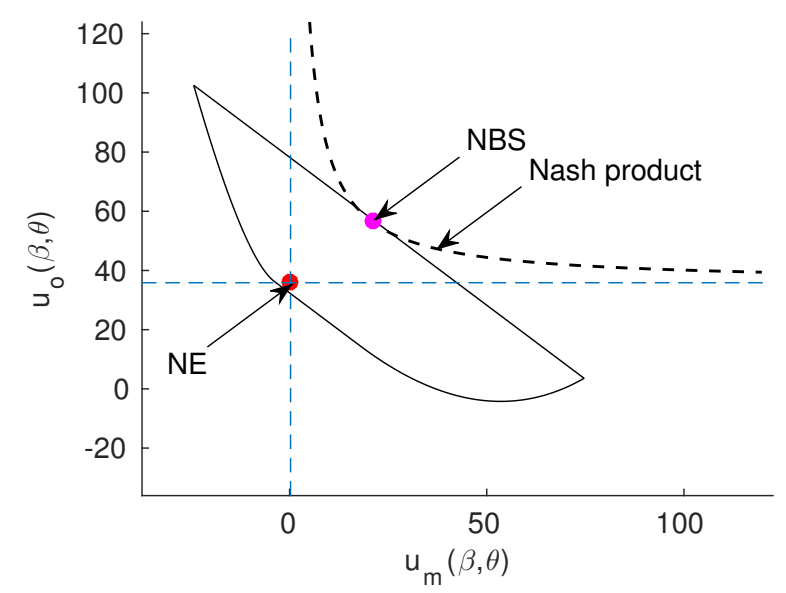

Fig. 1. The utility space for our model obtained from drawing a convex hull over utility pairs calculated for the action space of the MNO and OTT varied over $[0,1] \times[0,1]$, for a fixed clustering level and infrastructure price; marked points denote special operating points (NE - Nash Equilibrium, NBS - Nash Bargaining Solution); while the dashed curve corresponds to the Nash product curve.

breaks down. Obviously, this implies that if the cooperation provides lower payoff(s) to any one of the players, she will not be interested in cooperating, and will settle for the minimal utility. Often times it makes sense to choose as the disagreement point, the point related to the Nash equilibrium of the non-cooperative game. The Nash equilibrium corresponds to a set of strategies selected by players such that no individual player can increase her utility by unilaterally deviating.

Proposition 1: The Nash equilibrium strategy $\left(\beta_{\mathrm{NE}}, \theta_{\mathrm{NE}}\right)$ corresponds to the following pair of actions:

$$
\begin{aligned}
& \beta_{\mathrm{NE}}=1.0, \\
& \theta_{\mathrm{NE}}= \begin{cases}\frac{R-a_{1}}{2 a_{2}}, & \text { if } a_{2}>0, \\
\arg \max \left(u_{o}\left(\beta_{\mathrm{NE}}, 0.0\right), u_{o}\left(\beta_{\mathrm{NE}}, 1.0\right)\right), & \text { otherwise. }\end{cases}
\end{aligned}
$$

Proof: Let us start by observing that, given $\beta \in[0,1]$, the MNO has a dominant strategy $\beta_{\mathrm{NE}}=1.0$ as $u_{m}\left(\beta_{\mathrm{NE}}, \theta\right) \geq$ $u_{m}\left(\beta^{\prime}, \theta\right)$ for all $\beta^{\prime} \in[0,1]$ and $\theta \in[0,1]$. Given the dominant strategy of the MNO, we find the equilibrium strategy of the OTT by maximizing its utility, which leads to strategy $\theta_{\mathrm{NE}}=$ $\frac{R-a_{1}}{2 a_{2}}$, if $a_{2}>0$, or $\arg \max \left(u_{o}\left(\beta_{\mathrm{NE}}, 0.0\right), u_{o}\left(\beta_{\mathrm{NE}}, 1.0\right)\right)$ otherwise, which concludes the proof of the proposition.

The pair of outcomes in the disagreement point corresponds to the element $\left(u_{o}\left(\beta_{\mathrm{NE}}, \theta_{\mathrm{NE}}\right), u_{m}\left(\beta_{\mathrm{NE}}, \theta_{\mathrm{NE}}\right)\right)$. This disagreement point should be interpreted in the following way: a rational MNO has no incentive to set any other fee than $\beta_{\mathrm{NE}}$, therefore forcing an OTT to deploy either a full infrastructure or a significant portion of it.

\section{BARGAINING SOLUTION}

In the following we derive a formula that describes the Nash bargaining solution to our game. An NBS is the unique solution to the following optimization problem:

$$
\arg \max \prod_{i \in \mathcal{N}}\left(u_{i}(\beta, \theta)-d_{i}\right)
$$

subject to:

$$
\begin{aligned}
& u_{i}(\beta, \theta) \geq d_{i}, \quad \forall i \in \mathcal{N}, \\
& \beta, \theta \in[0,1],
\end{aligned}
$$

where $d_{i}=u_{i}\left(\beta_{\mathrm{NE}}, \theta_{\mathrm{NE}}\right)$ for each $i \in \mathcal{N}$. In this formulation constraints (4) ensure that our solution is no worse than the disagreement point, while constraint (5) ensures that the strategies available to the players are feasible.

Theorem 1: Given, the problem in (3), the NBS is:

$$
\left(\beta_{\mathrm{NBS}}, \theta_{\mathrm{NBS}}\right)=\left(\frac{Q-a_{0}+c_{m} b_{0}+d_{m}-d_{o}}{2 R}, 0.0\right) .
$$

Proof: Given $\Omega$, which is non-empty, compact and convex, we can find the maximum of (3) by first taking the logarithm of the product in (3) and then applying the Lagrangian multiplier method:

$$
\begin{aligned}
L(\beta, \theta, \mu, \eta, \gamma) & =\sum_{i \in \mathcal{N}} \log \left(u_{i}(\beta, \theta)-d_{i}\right)-\sum_{i \in \mathcal{N}} \mu_{i}\left(d_{i}-u_{i}(\beta, \theta)\right) \\
& +\eta_{o} \theta+\eta_{m} \beta-\gamma_{o}(\theta-1)-\gamma_{m}(\beta-1)
\end{aligned}
$$

where $\mu_{i} \geq 0, \eta_{i} \geq 0, \gamma_{i} \geq 0$. Then, the necessary and sufficient Karush-Kuhn-Tucker (KKT) conditions for optimality are:

$$
\begin{array}{r}
\nabla_{\beta} L(\beta, \theta, \mu, \eta, \gamma)=0, \\
\nabla_{\theta} L(\beta, \theta, \mu, \eta, \gamma)=0, \\
\mu_{i}\left(d_{i}-u_{i}(\beta, \theta)\right)=0, \quad \forall i \in \mathcal{N}, \\
-\eta_{o} \theta \leq 0,-\eta_{m} \beta \leq 0, \\
\gamma_{o}(\theta-1) \leq 0, \gamma_{m}(\beta-1) \leq 0,
\end{array}
$$

where (7) and (8) are the stationary conditions, and (9) and (10) are the complementary slackness conditions. After making substitutions and calculating the derivatives, the stationary conditions yield the following forms:

$$
\begin{array}{r}
\frac{-R(\theta-1)}{d_{o}-u_{o}(\beta, \theta)}+\frac{R(\theta-1)}{d_{m}-u_{m}(\beta, \theta)}-\gamma_{m}+\eta_{m}=0, \\
\frac{a_{1}-R \beta+2 a_{2} \theta}{d_{o}-u_{o}(\beta, \theta)}+\frac{R \beta+c_{m}\left(b_{1}+2 b_{2} \theta\right)}{d_{m}-u_{m}(\beta, \theta)}-\gamma_{m}+\eta_{m}=0 .
\end{array}
$$

When $\mu_{i}=0, \gamma_{i}=0, \eta_{m}=0$, and $\theta=0.0$, we get that $\beta=\left(Q-a_{0}+d_{m}-d_{o}+c_{m} b_{0}\right) /(2 R)$ and $\eta_{o}=\left(2\left(a_{1}+\right.\right.$ $\left.\left.b_{1} c_{m}\right)\right) /\left(a_{0}-Q+d_{m}+d_{o}+b_{0} c_{m}\right)$. This solution is not only feasible (provided that $Q-a_{0}+d_{m}-d_{o}+c_{m} b_{0}<2 R$ and $Q-a_{0}+d_{m}-d_{o}+c_{m} b_{0} \geq 0$ ), but it happens to lie on the Pareto frontier of our utility space, precisely at the intersection of the Nash product curve and the Pareto frontier of $\Omega$, as presented in Fig. 1. Any other feasible solutions will not lie on the Pareto frontier of $\Omega$, which concludes the proof.

Given Theorem 1, if the OTT and MNO cooperate, the OTT serves all of its demand through the infrastructure provided by the MNO and pays the traffic fee $\beta_{\mathrm{NBS}}$. In the following we will analyze the impact that the spatial distribution of demand and the infrastructure price have on this cooperation. 


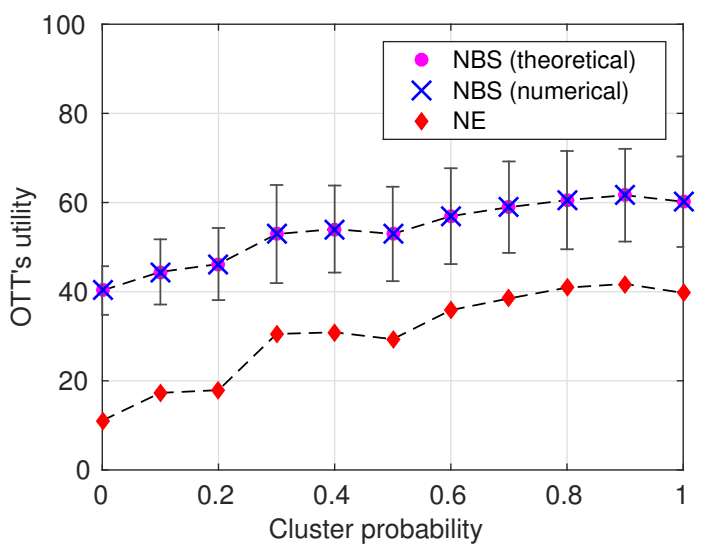

(a) OTT

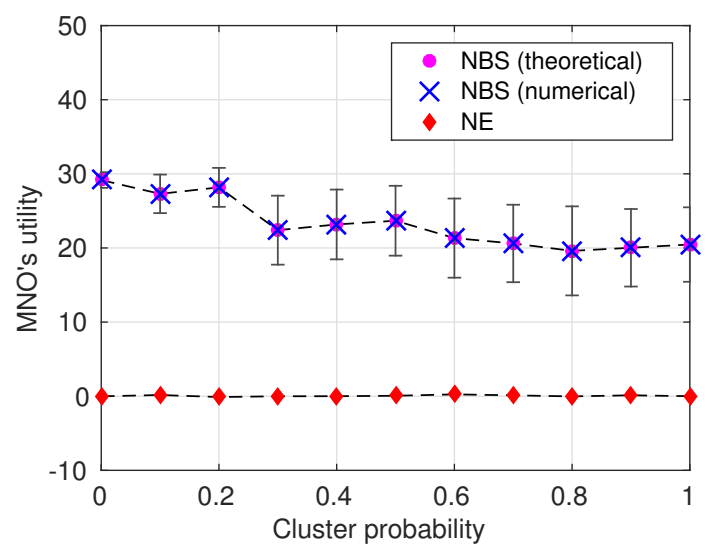

(b) MNO

Fig. 2. Utility obtained in the non-cooperative (NE) and cooperative (NBS) cases for different values of spatial clustering in the OTT's demand, with error bars accounting for the deviation in the number of base station required for a given cluster probability. When the cluster probability is close to 0.0 , the demand is almost uniformly distributed in space, whereas when the cluster probability approaches 1.0, the demand is generated from a number of highly concentrated clusters. The cost $c_{m}$ is fixed at 1.0 .

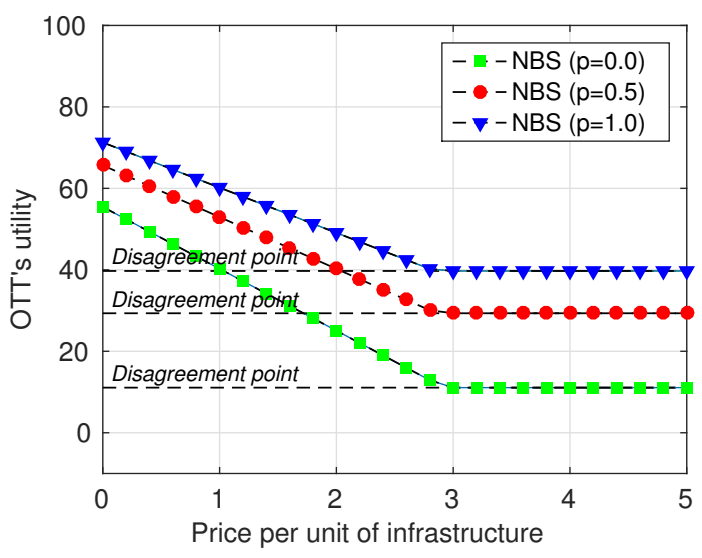

(a) OTT

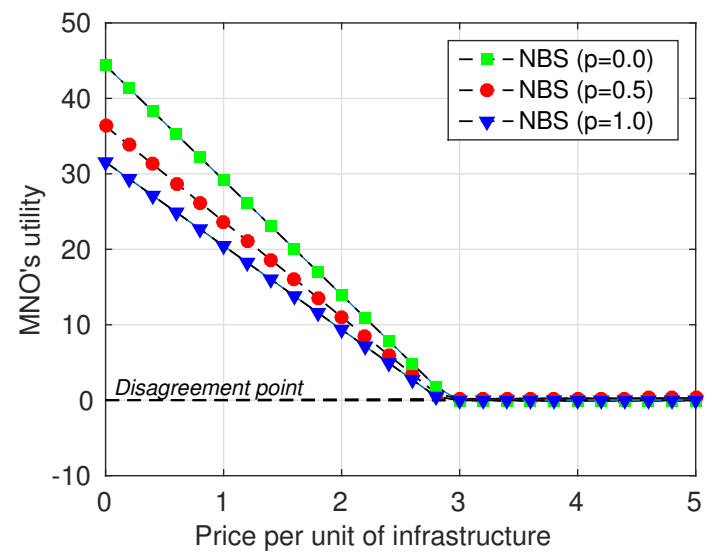

(b) MNO

Fig. 3. Utility obtained from the cooperation for different price points of the MNO's infrastructure, and three values of the cluster probability $p$. When $c_{m}<1.0$, the MNO's infrastructure is cheaper than the infrastructure deployed by the OTT. When $c_{m}>1.0$, the MNO pays more for the infrastructure than the OTT does. We see that both utilities are inversely proportional to the price of the MNO's infrastructure; yet, there is a price point at which the cooperation collapses to the disagreement point.

\section{NUMERICAL RESUlts AND DisCUSSION}

We validate our analytical solutions in Theorem 1 by applying to problem (3) iterative optimization methods, such as the sequential least square method. Indeed, in Fig. 2 and Fig. 3 we can see that the numerically obtained bargaining solutions match closely the ones expressed in closed-form, confirming the correctness of our derivations. In Table I we present estimates of the deployment function for various demand clustering levels. Let us recall Property 1: given these estimates, we can see that $a_{2}<0$, therefore for each clustering level the $\mathrm{NE}$ corresponds to the pair $\left(\beta_{\mathrm{NE}}, \theta_{\mathrm{NE}}\right)=(1.0,1.0)$, which means that the OTT fully deploys (or contracts the deployment of) its infrastructure to serve its demand. In our numerical analysis we also assume that the revenue obtained by the OTT, i.e., $Q$, is fixed and equal to the total volume of the demand $R$.

In Fig. 2 we show how the utility, obtained by the OTT (Fig. 2(a)) and MNO (Fig. 2(b)), changes with the clustering of the OTT's demand. What we can immediately note from
Fig. 2(a) is that the clustering of demand has a stronger impact on the utility obtained by the OTT. This has to do with the fact that clustering means less infrastructure is required to cover the same volume of demand, as we can observe in the NE case where the OTT deploys all the infrastructure on its own. Clearly, having to deploy less infrastructure per unit of demand makes deploying its own infrastructure a more attractive option for the OTT. To compensate for this fact an MNO has to decrease the traffic charge offered to the OTT, therefore giving up on some of its profits (even though the MNO will also have to deploy less infrastructure due to clustering of the demand).

The infrastructure cost $c_{m}$ has a significant impact on the cooperation between the MNO and the OTT. In Fig. 3(a) and Fig. 3(b) we observe that the utilities of the OTT and the MNO are inversely proportional to the infrastructure price, i.e., the higher the price, the lower the utility. The MNO compensates for higher infrastructure price by imposing a higher traffic fee on the OTT. When the price of MNO infrastructure becomes excessive, the cooperation is no longer feasible and the only 
TABLE I. ESTIMATES OF THE DEPLOYMENT FUNCTION FOR THE OTT AND MNO FOR VARYING LEVELS OF CLUSTERING IN DEMAND.

\begin{tabular}{ccc}
\hline & OTT & MNO \\
\hline Cluster probability & Parameters $\left\{a_{0}, a_{1}, a_{2}\right\}$ & Parameters $\left\{b_{0}, b_{1}, b_{2}\right\}$ \\
\hline 0.0 & $\{0.1,108.0,-12.9\}$ & $\{30.8,47.8,-17.4\}$ \\
0.1 & $\{-1.1,134.7,-53.6\}$ & $\{30.7,47.9,-17.3\}$ \\
0.2 & $\{-3.0,152.2,-84.4\}$ & $\{26.6,39.9,-13.1\}$ \\
0.3 & $\{-2.0,150.0,-80.9\}$ & $\{27.9,42.3,-14.2\}$ \\
0.4 & $\{-4.3,163.7,-100.2\}$ & $\{26.7,37.1,-10.4\}$ \\
0.5 & $\{-1.3,151.8,-88.5\}$ & $\{25.4,35.5,-10.2\}$ \\
0.6 & $\{-3.7,149.8,-88.1\}$ & $\{23.4,34.0,-10.5\}$ \\
0.7 & $\{-4.6,166.4,-109.2\}$ & $\{22.8,30.1,-6.8\}$ \\
0.8 & $\{-2.4,149.7,-81.4\}$ & $\{24.3,33.4,-9.1\}$ \\
0.9 & $\{-3.5,162.9,-99.6\}$ & $\{23.7,29.9,-5.9\}$ \\
1.0 & $\{-5.0,176.5,-129.3\}$ & $\{20.2,27.9,-8.0\}$ \\
\hline
\end{tabular}

reasonable solution for the players is to fall back to the disagreement point. The level of spatial clustering in the demand has little to no impact on the point where cooperation collapses.

Reflecting back on the achieved results, we can note two things. In real relationships between OTTs and MNOs, other considerations, besides cost, may influence the OTT's decision of whether to deploy its own infrastructure. If the expected monetary impact of these strategic considerations could be quantified, it would be possible to incorporate them into our model. On the MNO side, we do see that excessive cost of infrastructure, which in practice includes both the deployment and the spectrum licensing fees, may cause MNOs to be very protective over who can use their network and how. This may be comparable to the case of mobile virtual operators which were able to enter the European market only after favourable regulation was in place. However, falling revenues may be a motivator for MNOs to actually enter into deals with OTTs, as we can currently observe with Google Project Fi.

\section{CONCLUSION}

In this paper, we have analyzed a model of cooperation between an OTT (or a group of OTTs) and an MNO working together to deploy a wireless network crafted to the needs of a service offered by the OTT. For this model we have derived, using axiomatic bargaining, analytical terms expressing a rational bargaining solution. Our results show that efficient cooperation between an OTT and an MNO leads to the MNO solely deploying the radio access infrastructure and the OTT paying some reasonable traffic fee proportional to the volume of its demand. This cooperation is sustainable only if the cost of infrastructure to the MNO is not excessive. In case it significantly exceeds the price paid by the OTT for the infrastructure, cooperation is no longer feasible (the MNO is no longer able to increase the charge on the OTT, without the OTT retracting to its own deployment). In the non-cooperative case, the OTT deploys its own unlicensed infrastructure. Moreover, spatial clustering of the OTT demand means less radio access infrastructure is required to cover the same volume of demand. This means deploying its own infrastructure becomes cheaper for the OTT, and therefore more attractive. Effectively, it is the OTT that benefits the most from having spatially clustered demand.

\section{ACKNOWLEDGEMENT}

This material is based upon works supported by the Science Foundation Ireland under Grants No. 10/IN.1/I3007 and
10/CE/I1853.

\section{REFERENCES}

[1] N. Bhushan et al., "Network densification: the dominant theme for wireless evolution into 5G," IEEE Communications Magazine, vol. 52, no. 2, pp. 82-89, February 2014.

[2] S. Lawson. (2011, July) Survey: Wireless Networks Are Near Capacity. PC World. [Online]. Available: http://www.pcworld.com/ article/235964/wireless_networks_near_capacity.html

[3] L. Doyle et al., "Spectrum without Bounds, Networks without Borders," Proceedings of the IEEE, vol. 102, no. 3, pp. 351-365, March 2014.

[4] G. Owen, Game Theory. Academic Press, 1995.

[5] J. Kibiłda and L. A. DaSilva, "Efficient Coverage Through Interoperator Infrastructure Sharing in Mobile Networks," in Proc. of IFIP Wireless Days, November 2013, pp. 1-6.

[6] L. Cano et al., "Evaluating the performance of infrastructure sharing in mobile radio networks," in IEEE International Conference on Communications (ICC), June 2015, pp. 3222-3227.

[7] J. Monserrat et al., "Rethinking the mobile and wireless network architecture: The METIS research into 5G," in European Conference on Networks and Communications (EuCNC), June 2014, pp. 1-5.

[8] G. Landi et al., "SLA management and service composition of virtualized applications in mobile networking environments," in IEEE Network Operations and Management Symposium (NOMS), May 2014, pp. 1-8.

[9] J. Krämer, L. Wiewiorra, and C. Weinhardt, "Net neutrality: A progress report," Telecommunications Policy, vol. 37, no. 9, pp. 794-813, October 2013 .

[10] F. Fund, S. Hosseini, and S. Panwar, "More bars, more bang for the buck: Channel-dependent pricing for video delivery to mobile users," in IEEE Conference on Computer Communications (INFOCOM) Workshops, April 2014, pp. 565-570.

[11] E. Altman, M. K. Hanuwa, and R. Sundaresan, "Nonneutral network and the role of bargaining power in side payments," in Workshop on Network Control and Optimization (NET-COOP), November 2010.

[12] R. Ma and V. Misra, "The public option: a nonregulatory alternative to network neutrality," IEEE/ACM Transactions on Networking, vol. 21, no. 6, pp. 1866-1879, December 2013.

[13] G. Kesidis, "A simple two-sided market model with side-payments and ISP service classes," in IEEE Conference on Computer Communications (INFOCOM) Workshops, April 2014, pp. 595-597.

[14] J. Markendahl, "Mobile network operators and cooperation," Ph.D. dissertation, Royal Institute of Technology (KTH), 2011.

[15] E. Larsson and E. Jorswieck, "Competition Versus Cooperation on the MISO Interference Channel," IEEE Journal on Selected Areas in Communications, vol. 26, no. 7, pp. 1059-1069, September 2008.

[16] J. E. Suris et al., "Asymptotic Optimality for Distributed Spectrum Sharing Using Bargaining Solutions," IEEE Transactions on Wireless Communications, vol. 8, no. 10, pp. 5225-5237, October 2009.

[17] H. Boche et al., "Non-symmetric nash bargaining solution for resource allocation in wireless networks and connection to interference calculus," in Proc. of the 15th European Signal Processing Conference (EUSIPCO), September 2007, pp. 1317-1321.

[18] S.-L. Hew and L. B. White, "Cooperative Resource Allocation Games in Shared Networks: Symmetric and Asymmetric Fair Bargaining Models," IEEE Transactions on Wireless Communications, vol. 7, no. 11, pp. 4166-4175, November 2008.

[19] P. Lin et al., "Cooperation among wireless service providers: opportunity, challenge, and solution," IEEE Wireless Communications, vol. 17, no. 4, pp. 55-61, August 2010.

[20] J. Lempiainen and M. Manninen, UMTS Radio Network Planning, Optimization and Qos Management: For Practical Engineering Tasks. Kluwer Academic Publishers, 2004.

[21] E. Amaldi et al., "Optimization problems and models for planning cellular networks," in Handbook of optimization in telecommunications. Springer, 2006, pp. 917-939.

[22] M. Haenggi, Stochastic Geometry for Wireless Networks. Cambridge University Press, 2013. 